\title{
Evaluation of the relationship between Behcet's disease and cardiovascular disorders through metabolic function and copeptin
}

\author{
Senay Agirgol ${ }^{1}$, Evrim Cakir ${ }^{2}$, Alper Gumus 3 , Hatice Nur Ozturk ${ }^{4}$, Tugba Ozkok Akbulut ${ }^{1}$, Ozlem Turkoglu ${ }^{5}$, \\ Ilay Tasyurek ${ }^{1}$, Zafer Turkoglu ${ }^{1}$ \\ 'Department of Dermatology, Haseki Training and Education Hospital, Istanbul, Turkey \\ 2Department of Endocrinology and Metabolic Diseases, Haseki Training and Education Hospital, Istanbul, Turkey \\ ${ }^{3}$ Department of Clinical Chemistry, Haseki Training and Research Hospital, i̇stanbul, Turkey \\ ${ }^{4}$ Department of Dermatology, Bagcilar Training and Education Hospital, Istanbul, Turkey \\ ${ }^{5}$ Radiology Clinic, Kartal Training and Education Hospital, Istanbul, Turkey
}

Adv Dermatol Allergol 2019; XXXVI (5): 609-615 DOI: https://doi.org/10.5114/ada.2019.89509

\begin{abstract}
Introduction: Behcet's disease (BD) is a chronic inflammatory and multisystem vasculitis. Cardiac involvement is one of the major complications of BD. Cardiac involvement is sporadic in terms of its specific relationship to mortality. How to identify and follow up on cardiac-related complications in BD patients has yet to be determined.

Aim: The aim of our study is to assess cardiovascular diseases in BD patients by measuring metabolic function and copeptin levels and comparing these to a healthy control group. Knowing the specific metabolic functions that are negatively affected by BD will help doctors determine which functions need to be more closely monitored in BD patients. Our study is the first study in the available literature that evaluates copeptin in BD patients.

Material and methods: A control group of 58 healthy volunteers, including 32 females and 26 males (average age: $39.8 \pm 10.3$ years, range: $18-50$ years), was formed to compare with 84 BD patients (average age: $40.5 \pm 11$ years, range: 21-63 years), including 39 females and 45 males. Diastolic and systolic blood pressure, height, weight, body mass index (BMI), and waistline were measured for both groups. All study patients were also given hemograms, and fasting blood sugar (FBS), uric acid, lipid profile, insulin, C-reactive protein (CRP), and copeptin levels were measured. An ELISA Kit was used to measure copeptin.

Results: FBS, CRP, and insulin levels were significantly higher in the patient group $(p<0.001, p=0.004$, and $p=0.038$, respectively). Patients who had had the disease for more than 10 years had higher BMls, total cholesterol, low-density lipoprotein (LDL) cholesterol, and triglyceride (TG) levels $(p=0.034, p=0.004, p=0.016$, and $p=0.045$, respectively). Additionally, CRP levels were found to be higher in long-term patients and patients with active lesions. Diastolic blood pressure and waist circumference were also higher in the BD group. Patients who had active lesions had significantly higher diastolic blood pressure $(p=0.047)$. There were no statistically significant differences in copeptin levels between BD and control groups.

Conclusions: Cardiovascular involvement rarely contributes to the high mortality rate of BD patients. There were meaningful elevations in metabolic markers identified when BD and cardiovascular disease risk was assessed with metabolic parameters. The cardiac disease risk should be closely followed using metabolic functions, particularly in long-term BD patients with vascular involvement.
\end{abstract}

Key words: Behcet's disease, metabolic function, cardiovascular, copeptin.

\section{Introduction}

The incidence of Behcet's disease (BD) is between 1 and 70/100,000. It is most prevalent in Turkey and the Middle East, Japan, Korea, and China [1-4]. As most pa- tients are young adults, it might be assumed that BD patients would be in the low-risk group for cardiovascular disorders (CVD) [5]. Cardiac involvement is generally thought of as sporadic, yet it is one of the most serious complications of $\operatorname{BD}[5,6]$. Various cardiac manifestations

Address for correspondence: Senay Agirgol, Department of Dermatology, Haseki Training and Education Hospital, 34320 Istanbul, Turkey, phone: +90 50549871 14, e-mail: senayagirgol@hotmail.com Received: 8.12.2017, accepted: 15.06.2018. 
such as myocardial infarction, pericarditis, valve problems, aneurisms, or congestive cardiac failure have been reported. The nature, incidence, intensity, and management of cardiac involvement have not yet been clearly determined. Although many studies have been conducted on cardiac involvement and echo findings, there are fewer studies that evaluate the risks of cardiac disorders and the development of vascular damage in BD [6-8]. In this study we try to find specific metabolic functions that need to be monitored in BD patients.

Vascular inflammatory changes are the main changes seen in BD. Neutrophil or monocyte infiltration occurs around the blood vessels, and vascular inflammation affects the arteries, arterioles, capillaries, venules, and veins of different diameters. Chemotaxis, phagocytosis, superoxide, lysosomal enzyme production of hyperactive neutrophils, and increased CD11a and CD18 on the neutrophil walls have also been observed. Overreaction of neutrophils may cause tissue damage. Proinflammatory interleukin 1 (IL-1), IL-6, IL-8, and tumor necrosis factor $\alpha$ (TNF- $\alpha$ ) cytokines are increased in BD patients and are related to the activity of the disease [3, 4, 9-13]. It is thought that metabolic syndrome (MetS) develops due to psoriasis, $\mathrm{BD}$, and scleroderma patients even if coronary disease does not develop $[14,15]$.

Arginine vasopressin (AVP) is a neurohormone secreted by neurohypophysis that maintains homeostasis by reabsorbing water from the kidneys. Moreover, AVP affects osmoregulation and causes vasoconstriction. AVP plays a role in ACTH release, it affects glucose homeostasis through hepatic gluconeogenesis and glycogenolysis for lipid and glucose metabolisms. As extracellular glucose levels increase, insulin and glucagon release is also increased. Since AVP's half-life in the plasma is short, the C-terminal fragment of AVP's precursor, copeptin, is used to determine AVP levels [16].

\section{Aim}

The purpose of this study is to evaluate the CVD risk in BD patients based on metabolic function and copeptin levels. Moreover, the duration and activity of the disease will be examined.

\section{Material and methods}

Eigthy-four BD patients aged between 21 and 63, who sought treatment at the dermatology clinic of the Haseki Education and Training hospital, between March 2016 and December 2016 were included in the study. The International Study Group for Behcet's Disease criteria [17] were used to diagnose Behcet's Disease. The control group consisted of 58 healthy individuals of ages and genders similar to the BD group. Individuals with coronary artery disease, hypertension (HT) and diabetes mellitus (DM), renal failure, known valvular heart disorders, acute vascular involvement, malignity and additional inflammatory diseases, and those with acute or chronic infections were excluded from the study. Patients who have had the disease for at least 6 months were included in the study. Demographic characteristics of the BD and control groups (name, age, and gender) were recorded. The age when the disease first emerged, duration of the disease, and the medications used were also recorded. No cardiac symptoms were observed in the history of the patients or during the study. Arterial blood pressure, height, weight, body mass index (BMI), and waist circumference measurements were taken for both study groups. Fasting blood sugar (FBS), insulin, lipid levels, hemograms, CRP, and uric acid tests were measured in the patient and control groups, and copeptin levels were determined simultaneously after preserving the drawn blood at $-80^{\circ} \mathrm{C}$. Serum copeptin levels (Human Copeptin (CPP), ELISA Kit (Sunred Copeptin ELISA Kit, China) were measured using the sandwich test method. All patients were given informed consent forms, and the approval of the local ethics board was obtained.

\section{Statistical analysis}

SPSS 15.0 for Windows was used for statistical analysis. Defining statistics are presented as numbers and percentages for categorical variables, and they are shown as average, standard deviation (SD), and median for numeric variables. Comparison of numeric variables in 2 independent groups was carried out using the student's t-test when normal distribution was obtained and the Mann-Whitney $U$ test when normal distribution was not obtained. The relationships between numeric variables were examined using the Pearson correlation analysis when parametric test conditions were met and the Spearman correlation analysis when these conditions were not met. Statistical $\alpha$ significance level was assumed to be $p<0.05$.

\section{Results}

The patients were between 21 and 63 years of age $(40.5 \pm 11.1)$, and the average disease period was $10 \pm 7.5$ years (ranging between 1 and 40 years). The control group was between 21 and 63 years of age $(39.8 \pm 10.3)$. There were no statistically significant differences between the average ages and gender proportions of the patient and control groups. Fourty patients had had the disease for fewer than 10 years, and 44 had had it for 10 or more years (Table 1). While all of our patients used colchicine, some used prednisolone $(n=8)$, cyclosporine $(n=3)$, salicylate $(n=15)$, and azathioprine $(n=12)$. Four patients did not use colchicine regularly even if they had had the disease for more than 10 years. Eighteen patients in the study had serious involvement such as uveitis, deep vein thrombosis, and neuro-Behcet's disease, and 53 patients had active lesions. While there was no significant dif- 
Table 1. Demographic features of patient and control groups and comparison of DBP, SBP, disease severity and duration

\begin{tabular}{|c|c|c|c|c|c|}
\hline \multirow[t]{2}{*}{ Parameter } & \multicolumn{2}{|c|}{ Behçet group } & \multicolumn{2}{|c|}{ Control group } & \multirow[t]{2}{*}{$P$-value } \\
\hline & $n$ & $\%$ & $n$ & $\%$ & \\
\hline \multicolumn{6}{|l|}{ Gender: } \\
\hline Female & 45 & 53.6 & 32 & 55.2 & 0.851 \\
\hline Male & 39 & 46.4 & 26 & 44.8 & \\
\hline Parameter & $\begin{array}{c}\text { Mean } \pm \text { SD } \\
\text { or } n(\%)\end{array}$ & Median & Mean \pm SD & Median & $P$-value \\
\hline Age & $40.5 \pm 11.1$ & 41 & $39.8 \pm 10.3$ & 40 & 0.727 \\
\hline Initial age/age at onset & $30.1 \pm 11.0$ & 29 & & & \\
\hline Disease time [year]: & $10.4 \pm 7.5$ & 10 & & & \\
\hline$<10$ & \multicolumn{2}{|c|}{$40(47.6)$} & & & \\
\hline$\geq 10$ & \multicolumn{2}{|c|}{$44(52.4)$} & & & \\
\hline Active lesion & \multicolumn{2}{|c|}{$53(64.6)$} & & & \\
\hline Colchicine use [year]: & $9.0 \pm 6.1$ & 9 & & & \\
\hline$<10$ & \multicolumn{2}{|c|}{$46(54.7)$} & & & \\
\hline$\geq 10$ & \multicolumn{2}{|c|}{$38(45.3)$} & & & \\
\hline Severe disease & \multicolumn{2}{|c|}{$18(21.4)$} & & & \\
\hline SBP & $115.7 \pm 15.9$ & 110 & $116.4 \pm 18.5$ & 120 & 0.250 \\
\hline DBP & $74.9 \pm 9.1$ & 80 & $72.3 \pm 14.3$ & 70 & 0.012 \\
\hline Height & $164.4 \pm 8.8$ & 162 & $164.9 \pm 13.5$ & 164.5 & 0.376 \\
\hline Weight & $72.5 \pm 12.3$ & 71 & $72.2 \pm 13.0$ & 74 & 0.911 \\
\hline BMI & $26.9 \pm 4.5$ & 26.1 & $26.9 \pm 6.8$ & 26.0 & 0.651 \\
\hline WC & $91.8 \pm 12.3$ & 92 & $86.1 \pm 12.1$ & 87 & 0.007 \\
\hline
\end{tabular}

DBP - diastolic blood pressure, SBP - systolic blood pressure, WC - waist circumference.

ference between the BD and control groups in terms of height, weight, and BMI, diastolic blood pressure and waist circumference averages were statistically significantly higher in the patient group ( $p=0.012, p=0.007)$.

In addition to the high values of the laboratory parameters of FBS, insulin average, and CRP, the number of BD patients with CRP above 5 was significantly higher than in the control group $(p<0.001, p=0.004, p=0.038$, and $p=0.001$ ). There was no statistically significant difference between the two groups in terms of uric acid, lipid profile, hemograms, and copeptin values (Table 2).

Average BMI, cholesterol, low-density lipoprotein (LDL) cholesterol, and triglycerides (TG) in patients who had had the disease for more than 10 years were statistically significantly higher than in patients who had had BD for fewer than 10 years ( $p=0.034, p=0.004, p=$ 0.016 , and $p=0.045$ ) (Table 3 ). There was no statistically significant difference in terms of the other metabolic parameters.
The diastolic blood pressure of the patients with active lesions was statistically significantly higher than those without active lesions ( $p=0.047$ ) (Table 4).

The average FBS, uric acid, and triglyceride values of the patients with CRP levels above 5 were statistically significantly higher, and their average HDL and WBC were statistically significantly lower $(p=0.049$, $p=0.002, p=0.026, p=0.010, p=0.044$ ) (Table 5). The average copeptin level was $22.98 \mathrm{ng} / \mathrm{ml}$ (interquartile range: $1.45-22.98$ ) in the patient group and $19.67 \mathrm{ng} / \mathrm{ml}$ (0.31-19.98) in the control group. There were no statistically significant differences between the patient and control groups as to other study parameters.

\section{Discussion}

Behcet's disease is a vasculitis that involves perivascular tissue and blood vessel walls. It proceeds in recurrences and remissions. The oral mucosa, eyes, muscu- 
Table 2. Metabolic parameters and copeptin values of the patient and control groups

\begin{tabular}{|c|c|c|c|c|c|}
\hline \multirow[t]{2}{*}{ Parameter } & \multicolumn{2}{|c|}{ Patient } & \multicolumn{2}{|c|}{ Control } & \multirow[t]{2}{*}{$P$-value } \\
\hline & Mean \pm SD or $n(\%)$ & Median & Mean \pm SD or $n(\%)$ & Median & \\
\hline FBS & $97.3 \pm 22.6$ & 94 & $88.2 \pm 14.0$ & 89 & $<0.001$ \\
\hline Uric acid & $4.9 \pm 1.6$ & 4.6 & $4.9 \pm 1.3$ & 4.8 & 0.635 \\
\hline Cholesterol & $179.4 \pm 40.3$ & 179 & $186.4 \pm 38.1$ & 182 & 0.303 \\
\hline LDL & $109.5 \pm 32.0$ & 109.5 & $115.6 \pm 30.5$ & 113 & 0.261 \\
\hline HDL & $45.2 \pm 10.9$ & 45.5 & $48.6 \pm 13.0$ & 46.4 & 0.377 \\
\hline TG & $124.4 \pm 57.8$ & 112.5 & $118.2 \pm 67.8$ & 110 & 0.295 \\
\hline CRP: & $7.1 \pm 11.7$ & 3.3 & $2.8 \pm 3.2$ & 1.9 & 0.004 \\
\hline$\leq 5$ & \multicolumn{2}{|c|}{$53(63.9)$} & \multicolumn{2}{|c|}{$51(87.9)$} & 0.001 \\
\hline$>5$ & \multicolumn{2}{|c|}{$30(36.1)$} & \multicolumn{2}{|c|}{$7(12.1)$} & \\
\hline $\mathrm{Hgb}$ & $13.5 \pm 1.8$ & 13.7 & $13.3 \pm 2.0$ & 13.4 & 0.585 \\
\hline Hct & $40.1 \pm 6.5$ & 40.6 & $40.9 \pm 7.3$ & 39.8 & 0.427 \\
\hline PLT & $251.9 \pm 69.9$ & 247.5 & $246,7 \pm 59.8$ & 252.5 & 0.954 \\
\hline WBC & $7.9 \pm 8.6$ & 6.8 & $6.9 \pm 1.7$ & 6.71 & 0.878 \\
\hline Insulin & $8.0 \pm 5.1$ & 6.7 & $6.6 \pm 3.8$ & 5.83 & 0.038 \\
\hline Copeptin & $5.04 \pm 5.01$ & 2.85 & $4.62 \pm 5.12$ & 2.62 & 0.083 \\
\hline
\end{tabular}

FBS - fasting blood sugar, LDL - low-density lipoprotein cholesterol, HDL - high-density lipoprotein cholesterol, TG - triglycerides, Hgb - haemoglobin, Hct - haematocrit, PLT - platelets, WBC - white blood cells.

loskeletal system, lungs, gastrointestinal system, blood vessels, and neurologic system can be involved [1-4, 13]. Cardiovascular involvement has been reported in $7-46 \%$ of the cases. The mortality rate if there is large vessel involvement is around $20 \%$. Thrombosis and vasculitis with perivascular inflammation have been observed in involved arteries during biopsies and autopsies [8]. Some authors report that BD increases arterial hardness, and they argue that inflammation affects the artery structure and artery walls and increases the atherosclerotic process $[18,19]$.

It is not yet clear which cardiac disorders will affect BD patients and how these disorders should be monitored [20]. One meta-analysis suggests that BD causes diastolic dysfunction and congestive heart failure [8]. Thus, the disease needs to be closely monitored by cardiologists when additional risks are present. Previous studies show that insulin resistance is higher in BD patients compared to healthy individuals [21-24]. In our study, FBS, insulin levels, and diastolic blood pressure were statistically significantly higher in the BD group than in the control group. As the duration of the disease increased in the patient group, higher BMI, cholesterol, LDL cholesterol, triglycerides were observed in the patient group. High uric acid levels were observed in addition to high lipid levels in patients with high CRP levels. In Şahin et al.'s study, while high levels of CRP were observed in
BD patients, no differences were observed in terms of cholesterol values and insulin [22].

Copeptin is used to indicate the severity and progression of the disease in cardiac failure. More recently, copeptin was recognized as a good biomarker of MetS $[16,25]$. In several studies, copeptin was shown to be associated with insulin resistance, obesity, and metabolic disturbances. Our study is the first study in the available literature which evaluates copeptin in BD patients. The fact that our study did not observe a statistically significant elevation of copeptin between the two groups may be due to the fact that patients with cardiovascular disease and acute vascular damage were excluded from our study, so the participating patients had not yet reached the level of cardiac failure.

Cardiac involvement in BD patients is reported sporadically, and so is for patients who do not use regular treatment. One 65-year-old female patient developed $\mathrm{AMI}$ and pulmonary oedema, and this case exhibited mitral valve and aorta regurgitation in the echo, and aneurisms in the follow-up [20]. An 80-year-old male patient reported coronary infarction, and a 12-year-old male patient reported acute myocardial infarction. The provided explanation was that local coronary vasculitis increases intimal fibrosis and causes coronary occlusion, leading to acute coronary syndrome, acute myocardial infarction, or unstable angina. It was argued that the disruption of mi- 
Table 3. Evaluation of the metabolic parameters of patients who had had the disease for more than 10 years and less than 10 years

\begin{tabular}{|c|c|c|c|c|c|c|c|}
\hline \multirow[t]{3}{*}{ Parameter } & \multicolumn{6}{|c|}{ Disease time } & \multirow[t]{3}{*}{$P$-value } \\
\hline & \multicolumn{3}{|c|}{$<10$} & \multicolumn{3}{|c|}{$\geq 10$} & \\
\hline & Mean & SD & Median & Mean & SD & Median & \\
\hline Systolic blood pressure & 116.8 & 15.1 & 115 & 114.7 & 16.6 & 110 & 0.464 \\
\hline Diastolic blood pressure & 75.3 & 9.1 & 80 & 74.5 & 9.3 & 75 & 0.735 \\
\hline BMI & 25.7 & 3.7 & 24.4 & 27.9 & 5.0 & 27.9 & 0.034 \\
\hline Waist circumference & 89.9 & 12.1 & 88.5 & 93.6 & 12.4 & 93 & 0.166 \\
\hline FBS & 97.6 & 30.3 & 92.5 & 97.0 & 12.5 & 94 & 0.249 \\
\hline Uric acid & 4.7 & 1.4 & 4.5 & 5.0 & 1.8 & 4.7 & 0.301 \\
\hline Cholesterol & 166.4 & 37.4 & 163 & 191.2 & 39.5 & 186.5 & 0.004 \\
\hline LDL & 100.8 & 29.4 & 99 & 117.5 & 32.5 & 115 & 0.016 \\
\hline HDL & 43.4 & 12.5 & 44.8 & 46.9 & 9.0 & 46.45 & 0.140 \\
\hline TG & 114.9 & 60.3 & 100 & 133.1 & 54.6 & 120 & 0.045 \\
\hline CRP & 8.9 & 15.4 & 2.9 & 5.4 & 6.3 & 3.26 & 0.715 \\
\hline $\mathrm{Hgb}$ & 13.4 & 1.8 & 13.6 & 13.6 & 1.7 & 13.75 & 0.531 \\
\hline Hct & 38.8 & 8.6 & 40.2 & 41.3 & 3.5 & 41 & 0.316 \\
\hline PLT & 247.6 & 67.2 & 245.5 & 255.8 & 72.9 & 248.5 & 0.400 \\
\hline WBC & 9.3 & 12.3 & 7.1 & 6.6 & 1.6 & 6.5 & 0.054 \\
\hline Insulin & 7.8 & 5.1 & 6.8 & 8.2 & 5.3 & 6.6 & 0.956 \\
\hline Copeptin & 4.96 & 4.53 & 2.94 & 5.12 & 5.46 & 2.66 & 0.295 \\
\hline
\end{tabular}

FBS - fasting blood sugar, LDL - low-density lipoprotein cholesterol, HDL - high-density lipoprotein cholesterol, TG - triglycerides, Hgb - haemoglobin, Hct - haematocrit, PLT - platelets, WBC - white blood cells.

Table 4. Comparison of blood pressures of patients with active and inactive disease lesions

\begin{tabular}{|c|c|c|c|c|c|c|c|}
\hline \multirow[t]{3}{*}{ Blood pressure } & \multicolumn{6}{|c|}{ Active lesion } & \multirow[t]{3}{*}{$P$-value } \\
\hline & \multicolumn{3}{|c|}{ No } & \multicolumn{3}{|c|}{ Yes } & \\
\hline & Mean & SD & Median & Mean & SD & Median & \\
\hline Systolic & 113.8 & 16.3 & 110 & 117.1 & 15.8 & 110 & 0.507 \\
\hline Diastolic & 72.4 & 9.5 & 70 & 76.6 & 8.5 & 80 & 0.047 \\
\hline
\end{tabular}

crovascular function causes coronary incidents [26-31]. When our study was taking place, a 31-year-old patient who had not done any follow-up for 12 years was hospitalized with vena cava superior syndrome, and 2 patients were hospitalized with deep ulcers after deep venous thrombosis. These patients were excluded from our study due to the presence of acute vascular disorders.

There are several studies that report various opinions on ventricular function disruptions in BD patients. While studies that report no symptomatic systolic function disorders, or when right or left ventricle systolic function involvement exists, some studies report left ventricle diastolic function disorders. In line with these studies, diastolic blood pressure was statistically higher in the patient group, particularly in the long-term involvement group. Our results contribute to the view that diastolic function is involved in BD patients [8]. We believe that BD patients with vasculitis and existing inflammation are in the highrisk group for cardiac disorders and MetS.

Low-level inflammation with proinflammatory cytokines and insulin causes MetS [22]. BD is a chronic inflammatory disease in which cytokines such as TNF- $\alpha$, 
Table 5. Comparison of blood pressures, waist circumferences and metabolic parameters of patients with high and normal CRP levels

\begin{tabular}{|c|c|c|c|c|c|c|c|}
\hline \multirow[t]{3}{*}{ Parameter } & \multicolumn{6}{|c|}{ CRP } & \multirow[t]{3}{*}{$P$-value } \\
\hline & \multicolumn{3}{|c|}{$\leq 5$} & \multicolumn{3}{|c|}{$>5$} & \\
\hline & Mean & SD & Median & Mean & SD & Median & \\
\hline Systolic blood pressure & 116.1 & 16.4 & 110 & 115.0 & 15.3 & 110 & 0.843 \\
\hline Diastolic blood pressure & 76.0 & 9.1 & 80 & 73.0 & 9.2 & 70 & 0.215 \\
\hline BMI & 26.4 & 4.5 & 25.2 & 27.9 & 4.6 & 28.0 & 0.154 \\
\hline Waist circumference & 90.9 & 11.1 & 92 & 94.0 & 13.8 & 94.5 & 0.267 \\
\hline FBS & 93.7 & 10.3 & 92 & 103.4 & 34.8 & 97 & 0.049 \\
\hline Uric acid & 4.5 & 1.1 & 4.4 & 5.6 & 2.0 & 5.2 & 0.002 \\
\hline $\mathrm{HDL}$ & 47.4 & 10.1 & 46.7 & 41.1 & 11.3 & 42.35 & 0.010 \\
\hline TG & 114.6 & 53.2 & 106 & 143.1 & 62.5 & 135 & 0.026 \\
\hline WBC & 8.3 & 10.8 & 6.6 & 7.3 & 1.5 & 7.4 & 0.044 \\
\hline
\end{tabular}

BMI - body mass index, FBS - fasting blood sugar, HDL - high-density lipoprotein cholesterol, TG - triglycerides, WBC - white blood cells.

IL-1, and IL-6 are involved. In a study in which BD and MetS were evaluated, MetS was observed 2.67 times more, and it was observed more frequently in the patient group over the age of 40 . In this study, patients with GIS and neurologic involvement were assessed to have a high risk of MetS [31]. Patients who had had BD for more than 10 years were considered to be at risk in terms of metabolic functions, even when they were under 40 years of age. These serious effects of BD are more common in males. In female patients, hypertension and MetS are more common; increased physical activity, and close monitoring for hyperlipidaemia, diabetes, and hypertension are recommended for these patients [30]. There was no statistically significant difference in terms of serious vascular involvement and metabolic function between male and female patients in our study. The addition of metabolic function to this vasculitic inflammation suggests that $B D$ is a serious cardiac risk.

Colchicine has been used for a long time to treat neutrophilic disorders such as BD, FMF, and gout [32, 33]. Colchicine suppresses the chemotactic activity of neutrophils, which in turn suppresses mucocutaneous and vascular complications. The cardiovascular effects of the drug are being investigated.

It has been shown that colchicine used in doses between $0.5 \mathrm{mg}$ and $2.5 \mathrm{mg}$ for neutrophilic rheumatologic disorders cured pericarditis, myocardial ischemia, and coronary occlusion. It reduces CRP to a level under $2 \mathrm{mg} / \mathrm{dl}$, decreases myocardial damage, and maintains atrial and ventricular impulse generation. Since all of our patients used colchicine regularly and there were no patients who had had the disease for a long period and who did not use colchicine, the groups could not be compared. However, many case studies on BD patients with cardiac involvement reported that these patients benefit from immunosuppressive treatment and exhibit a good prognosis [33]. Gasparyan et al. argue that colchicine has a protective effect against atherosclerosis when low cholesterol levels are maintained [34].

\section{Conlusions}

$\mathrm{BD}$ is a disease in which the atherosclerotic process is accelerated, which exhibits as chronic inflammation and vasculitic findings in blood vessel walls. As BD patients are at risk of developing cardiovascular disorders, we believe that these patients must be closely monitored based on metabolic parameters for cardiovascular disorder. We believe that the early diagnosis and prevention of cardiovascular disorders in BD patients require a multidisciplinary approach.

\section{Conflict of interest}

The authors declare no conflict of interest.

\section{References}

1. Farouk H, Zayed HS, El-Chilali K. Cardiac findings in patients with Behcet's disease: facts and controversies. Anatol I Cardiol 2016; 16: 529-33.

2. Azizlerli G, Kose AA, Sarica R, et al. Prevalence of Behcet's disease in Istanbul, Turkey. Int I Dermatol 2003; 42: 803-6.

3. Alpsoy E. Behcet's disease: a comprehensive review with a focus on epidemiology, etiology and clinical features, and management of mucocutaneous lesions. J Dermatol 2016; 43: 620-32. 
4. Davatchi F, Chams-Davatchi C, Shams H, et al. Behcet's disease: epidemiology, clinical manifestations, and diagnosis. Expert Rev Clin Immunol 2017; 13: 57-65.

5. Gurler A, Boyvat A, Tursen U. Clinical manifestations of Behcet's disease: an analysis of 2147 patients. Yonsei Med J 1997; 38: 423-7.

6. Aslam F, Bandeali SJ, Crowson C, Alam M. Cardiac function and diastolic dysfunction in behcet's disease: a systematic review and meta-analysis. Int J Rheumatol 2016; 2016: 9837184.

7. Heper G, Polat M, Yetkin E, Senen K. Cardiac findings in Behcet's patients. Int J Dermatol 2010; 49: 574-8.

8. Geri G, Wechsler B, Thi Huong du L, et al. Spectrum of cardiac lesions in Behcet disease: a series of 52 patients and review of the literature. Medicine (Baltimore) 2012; 91: 25-34.

9. Rajendram R, Rao NA. Molecular mechanisms in Behcet's disease. Br J Ophthalmol 2003; 87: 1199-200.

10. Alayli G, Aydin F, Coban AY, et al. T helper 1 type cytokines polymorphisms: association with susceptibility to Behcet's disease. Clin Rheumatol 2007; 26: 1299-305.

11. Adam B, Calikoglu E. Serum interleukin-6, procalcitonin and C-reactive protein levels in subjects with active Behcet's disease. J Eur Acad Dermatol Venereol 2004; 18: 318-20.

12. Yalcindag FN, Yalcindag A, Batioglu F, et al. Evaluation of serum resistin levels in patients with ocular and non-ocular Behcet's disease. Can J Ophthalmol 2008; 43: 473-5.

13. Baggiolini M, Moser B, Clark-Lewis I. Interleukin-8 and related chemotactic cytokines. The Giles Filley Lecture. Chest 1994; 105: 95S-8S.

14. La Montagna G, Cacciapuoti F, Buono R, et al. Insulin resistance is an independent risk factor for atherosclerosis in rheumatoid arthritis. Diab Vasc Dis Res 2007; 4: 130-5.

15. El Magadmi M, Ahmad Y, Turkie W, et al. Hyperinsulinemia, insulin resistance, and circulating oxidized low density lipoprotein in women with systemic lupus erythematosus. J Rheumatol 2006; 33: 50-6.

16. Bolignano D, Cabassi A, Fiaccadori E, et al. Copeptin (CTproAVP), a new tool for understanding the role of vasopressin in pathophysiology. Clin Chem Lab Med 2014; 52: 1447-56.

17. Criteria for diagnosis of Behcet's disease. International Study Group for Behcet's Disease. Lancet 1990; 335: 1078-80.

18. Ikonomidis I, Lekakis J, Stamatelopoulos K, et al. Aortic elastic properties and left ventricular diastolic function in patients with Adamantiades-Behcet's disease. J Am Coll Cardiol 2004; 43: 1075-81.

19. Tunc SE, Dogan A, Gedikli O, et al. Assessment of aortic stiffness and ventricular diastolic functions in patients with Behcet's disease. Rheumatol Int 2005; 25: 447-51.

20. Veilleux SP, O'Connor K, Couture C, et al. What the cardiologist should know about cardiac involvement in Behcet disease. Can J Cardiol 2015; 31: 1485-8.

21. Kim SK, Choe JY, Park SH, et al. Increased insulin resistance and serum resistin in Korean patients with Behcet's disease. Arch Med Res 2010; 41: 269-74.

22. Sahin E, Karaman G, Uslu M, et al. Adiponectin levels, insulin resistance and their relationship with serum levels of inflammatory cytokines in patients with Behcet's disease. J Eur Acad Dermatol Venereol 2012; 26: 1498-502.

23. Erdem H, Dinc A, Pay S, et al. Peripheral insulin resistance in patients with Behcet's disease. J Eur Acad Dermatol Venereol 2006; 20: 391-5.
24. Capkin E, Karkucak M, Kola M, et al. Ischemia-modified albumin (IMA): a novel marker of vascular involvement in Behcet's disease? Joint Bone Spine 2015; 82: 68-9.

25. Melander O. Vasopressin, from regulator to disease predictor for diabetes and cardiometabolic risk. Ann Nutr Metab 2016; 68 Suppl 2: 24-8.

26. Kosar F, Sahin I, Gullu H, Cehreli S. Acute myocardial infarction with normal coronary arteries in a young man with the Behcet's disease. Int I Cardiol 2005; 99: 355-7.

27. Zhuang J, Wang S, Zhang Z, et al. Acute myocardial infarction and ascending aortic aneurysm in a child with Behcet's disease. Turk J Pediatr 2008; 50: 81-5.

28. Louali FE, Tamdy A, Soufiani A, et al. Cardiac thrombosis as a manifestation of Behcet syndrome. Tex Heart Inst J 2010; 37: 568-71.

29. Demirelli S, Degirmenci H, Inci S, Arisoy A. Cardiac manifestations in Behcet's disease. Intractable Rare Dis Res 2015; 4: 70-5.

30. Yalcin B, Gur G, Artuz F, Alli N. Prevalence of metabolic syndrome in Behcet disease: a case-control study in Turkey. Am J Clin Dermatol 2013; 14: 421-5.

31. Yahalom M, Bloch L, Suleiman K, et al. Cardiovascular involvement in Behcet disease: clinical implications. Int J Angiol 2016; 25: e84-e6.

32. Matsumura N, Mizushima Y. Leucocyte movement and colchicine treatment in Behcet's disease. Lancet 1975; 2: 813.

33. Lisitsyna T, Alekberova Z, Ovcharov P, et al. Left ventricular intracardiac thrombus in a patient with Behcet disease successfully treated with immunosuppressive agents without anticoagulation: a case report and review of the literature. Rheumatol Int 2015; 35: 1931-5.

34. Gasparyan AY, Ayvazyan L, Yessirkepov M, Kitas GD. Colchicine as an anti-inflammatory and cardioprotective agent. Expert Opin Drug Metab Toxicol 2015; 11: 1781-94. 